\title{
NORMS OF BASIC ELEMENTARY OPERATORS ON ALGEBRAS OF REGULAR OPERATORS
}

\author{
A. W. WICKSTEAD
}

(Communicated by Thomas Schlumprecht)

\begin{abstract}
We show that if $E$ is an atomic Banach lattice with an order continuous norm, $A, B \in \mathcal{L}^{r}(E)$ and $M_{A, B}$ is the operator on $\mathcal{L}^{r}(E)$ defined by $M_{A, B}(T)=A T B$, then $\left\|M_{A, B}\right\|_{r}=\|A\|_{r}\|B\|_{r}$ but that there is no real $\alpha>0$ such that $\left\|M_{A, B}\right\| \geq \alpha\|A\|_{r}\|B\|_{r}$.
\end{abstract}

\section{INTRODUCTION}

An elementary operator on a Banach algebra $\mathcal{A}$ is an operator of the form $T \mapsto$ $\sum_{k=1}^{n} A_{k} T B_{k}$, where each $A_{k}, B_{k} \in \mathcal{A}$. If $n=1$ such an elementary operator is called basic. There is substantial literature about elementary operators, much of it in the setting of $C^{*}$-algebras, and there are also significant results about the norm in the case of the algebra $\mathcal{L}(E)$ of all bounded operators on a Banach space $E$. The elementary starting point for such results in this case is the fact, which is a simple consequence of the Hahn-Banach theorem, that the norm of a basic elementary operator $T \mapsto A T B$ is precisely $\|A\|\|B\|$. This statement refers, of course, to the norm of $T \mapsto A T B$ as an element of the space of bounded operators on $\mathcal{L}(E)$. In this note, we look at the corresponding question for the space of regular operators $\mathcal{L}^{r}(E)$ on a Dedekind complete Banach lattice $E$. This is both a Banach lattice (for the usual order for operators between Banach lattices) and a Banach algebra under composition provided it is equipped with the regular norm $\|T\|_{r}=\||T|\|$. As $T \mapsto A T B$ defines elements of both $\mathcal{L}\left(\mathcal{L}^{r}(E)\right)$ and $\mathcal{L}^{r}\left(\mathcal{L}^{r}(E)\right)$, this means that we can ask about both its regular and operator norms. We will see that, at least in the slightly specialized setting in which we are able to obtain satisfactory results, there are rather different answers in the two cases. This is in contrast to the only related result in the literature, Proposition 1 of [6], that this author knows of.

Our interest in this problem is not purely for the sake of generalization. For $A_{k}, B_{k} \in Z(E)$, the centre of $E$ (the order ideal generated by the identity on $E$ ), operators of the form $T \mapsto \sum_{k=1}^{n} A_{k} T B_{k}$ have already featured in the literature in connection with descriptions of the centre of $\mathcal{L}^{r}(E)$. The interested reader is referred to [1, 2] and [9] for details, albeit in slightly more general settings.

We refer the reader to 5 . for the standard terminology in the field of Banach lattices and linear operators on them.

Received by the editors March 26, 2014 and, in revised form, May 6, 2014 and September 18, 2014.

2010 Mathematics Subject Classification. Primary 47B48, 47B60.

Key words and phrases. Regular operators, basic elementary operators, Banach lattices. 


\section{Notation AND History}

We will always assume that $E$ is a Dedekind complete Banach lattice, so that $\mathcal{L}^{r}(E)$ is also a Dedekind complete Banach lattice (and Banach algebra) under the regular norm. If $A, B \in \mathcal{L}^{r}(E)$, then we will denote by $M_{A, B}$ the operator $T \mapsto A T B$ which is easily seen to map $\mathcal{L}^{r}(E)$ into itself and to be an element of both $\mathcal{L}\left(\mathcal{L}^{r}(E)\right)$ and of $\mathcal{L}^{r}\left(\mathcal{L}^{r}(E)\right)$. A priori there is no reason to expect the two norms $\left\|M_{A, B}\right\|$ and $\left\|M_{A, B}\right\|_{r}$ to be the same, but what historical results exist might lead one to believe in such a possibility.

In [6], Schep studied the left regular representation of $\mathcal{L}^{r}(E)$ which, in our notation, is the mapping $A \mapsto M_{A, I}$ where $I$ denotes the identity operator on $E$. In his Proposition 1, he establishes that $\left\|M_{A, I}\right\|=\left\|M_{A, I}\right\|_{r}=\|A\|_{r}$. After that result he also remarks that $\left\|M_{I, B}\right\|=\left\|M_{I, B}\right\|_{r}=\|B\|_{r}$. As part of his Proposition 1, he also establishes the rather significant fact that $\left|M_{A, I}\right|=M_{|A|, I}$. Rather earlier, in Satz 3.1 (a) of [8], Synnatzschke proved the slightly more general result that $\left|M_{A, B}\right|=M_{|A|, B}$ whenever $B$ is positive. In the remarks following Proposition 1 , Schep leaves open the possibility that $\left|M_{I, B}\right|=M_{I,|B|}$. We must leave open the same question, but do wish to point out that this is obviously true when $E$ is reflexive. This is because the mapping $T \mapsto T^{*}: \mathcal{L}^{r}(E) \rightarrow \mathcal{L}^{r}\left(E^{*}\right)$ is a linear order isomorphism and $M_{I, B}(T)^{*}=M_{B^{*}, I^{*}}\left(T^{*}\right)$. We feel that it should be true that $\left|M_{I, B}\right|=M_{I,|B|}$ at least when $E$ has an order continuous norm. Satz 3.1 (b) of $[8]$ is relevant here.

\section{THE REGULAR NORM}

Our results for the regular norm of $M_{A, B}$ are restricted to the setting that $E$ is atomic with an order continuous norm. Such Banach lattices are certainly Dedekind complete. The reader will not go too far astray in thinking of $E$ as being something like $\ell_{p}$ for $1 \leq p<\infty$.

One reason that we are able to obtain results for such spaces is the observation that if $e$ is an atom of $E$ and $T \in \mathcal{L}^{r}(E)$, then $|T|(e)=|T(e)|$. This follows from the well known formula $|T|(e)=\bigvee\{T(x):|x| \leq e\}$. As $e$ is an atom, the $x$ 's here are of the form $\lambda e$ for $\lambda \in[-1,1]$ so that $|T|(e)=\bigvee\{\mid \lambda T(e): \lambda \in[-1,1]\}=|T(e)|$. If $f \in E^{*}$ and $x \in E$, then the operator $T=f \otimes x: e \mapsto f(e) x$ is certainly a member of $\mathcal{L}^{r}(E)$. Furthermore, $M_{A, B}(T)(e)=A(T(B(e)))=A(f(B(e)) x)=$ $f(B(e)) A(x)$, for any $e \in E$.

Theorem 3.1. If $E$ is an atomic Banach lattice with an order continuous norm and $A, B \in \mathcal{L}^{r}(E)$, then $\left|M_{A, B}\right|=M_{|A|,|B|}$.

Proof. Suppose that $T \in \mathcal{L}^{r}(E)_{+}$. As products of positive operators are positive, decomposing $A$ and $B$ into their positive and negative parts we have

$$
\begin{aligned}
M_{A, B}(T)=A T B & =\left(A_{+}-A_{-}\right) T\left(B_{+}-B_{-}\right) \\
& =A_{+} T B_{+}-A_{+} T B_{-}-A_{-} T B_{+}+A_{+} T B_{+} \\
& \leq A_{+} T B_{+}+A_{+} T B_{-}+A_{-} T B_{+}+A_{+} T B_{+} \\
& =\left(A_{+}+A_{-}\right) T\left(B_{+}+B_{-}\right) \\
& =|A| T|B|=M_{|A|,|B|}(T),
\end{aligned}
$$

which shows that $\left|M_{A, B}\right| \leq M_{|A|,|B|}$. 
We establish the reverse inequality first for the particular case that $T=f \otimes x$ where $f \in E_{+}^{*}$ and $x \in E_{+}$. As non-negative linear combinations of the atoms in $E$ are norm dense in the positive cone of $E$, it suffices to prove that $\left|M_{A, B}\right|(f \otimes x)(e) \geq$ $M_{|A|,|B|}(f \otimes x)(e)$ for any atom $e \in E_{+}$. Note that

$$
\begin{aligned}
\left|M_{A, B}\right|(f \otimes x) & =\bigvee\left\{M_{A, B}(S):|S| \leq f \otimes x\right\} \\
& \geq \bigvee\left\{M_{A, B}(g \otimes y):|y| \leq x,|g| \leq f\right\},
\end{aligned}
$$

so that

$$
\begin{aligned}
\left|M_{A, B}\right|(f \otimes x)(e) & \geq \bigvee\left\{\left|M_{A, B}(g \otimes y)\right|(e):|y| \leq x,|g| \leq f\right\} \\
& =\bigvee\left\{\left|M_{A, B}(g \otimes y)(e)\right|:|y| \leq x,|g| \leq f\right\} \\
& =\bigvee\{|g(B e) A y|:|y| \leq x,|g| \leq f\} \\
& =\bigvee\{|g(B e)||A y|:|y| \leq x,|g| \leq f\} \\
& =\bigvee\{|g(B e)|:|g| \leq f\} \bigvee\{|A y|:|y| \leq x\} \\
& =f(|B e|)|A|(x) \\
& =f(|B| e)|A|(x) \quad \text { as } e \text { is an atom } \\
& =M_{|A|,|B|}(f \otimes x)(e) .
\end{aligned}
$$

We have now established that $\left|M_{A, B}\right|(f \otimes x)=M_{|A|,|B|}(f \otimes x)$, if $f \in E_{+}^{*}$ and $x \in E_{+}$. By linearity this will also hold for any $f \in E^{*}$ and $x \in E$ and therefore $\left|M_{A, B}\right|(T)=M_{|A|,|B|}(T)$ for any finite rank operator $T$ on $E$.

Now take any $T \in \mathcal{L}^{r}(E), F$ a finite set of atoms in $E$ and $P_{F}$ the band projection onto the linear span of these atoms. As $E$ is atomic with an order continuous norm, $P_{F}(x) \rightarrow x$ in norm as the sets $F$ increase. In particular, this means that the finite rank operators $P_{F} \circ T$ converge upwards to $T$ for the strong operator topology. It follows easily that $M_{|A|,|B|}\left(P_{F} \circ T\right)$ converges strongly to $M_{|A|,|B|}(T)$. On the other hand if $T \geq 0$ and $x \in E_{+}$, then we have

$$
0 \leq\left|M_{A, B}\right|\left(T-P_{F} \circ T\right)(x) \leq M_{|A|,|B|}\left(T-P_{F} \circ T\right)(x) \rightarrow 0 .
$$

Hence $\left|M_{A, B}\right|\left(T-P_{F} \circ T\right)(x) \rightarrow 0$ from which it follows that $\left|M_{A, B}\right|\left(P_{F} \circ T\right)$ $\rightarrow\left|M_{A, B}\right|(T)$ strongly for positive $T$ and hence for all regular $T$. From these observations it is immediate that $\left|M_{A, B}\right|(T)=M_{|A|,|B|}(T)$ for all $T \in \mathcal{L}^{r}(E)$ and hence that $\left|M_{A, B}\right|=M_{|A|,|B|}$.

Corollary 3.2. If $E$ is an atomic Banach lattice with an order continuous norm and $A, B \in \mathcal{L}^{r}(E)$, then $\left\|M_{A, B}\right\|_{r}=\|A\|_{r}\|B\|_{r}$.

Proof. We certainly always have $\left\|M_{A, B}\right\|_{r} \leq\|A\|_{r}\|B\|_{r}$. If $A$ and $B$ are positive, then, as in the Banach space case, we have $\left\|M_{A, B}\right\|=\|A\|\|B\|$. To see this take $\epsilon>0$ and pick $x \in E_{+}$with $\|x\|=1$ and $\|A(x)\| \geq\|A\|-\epsilon$, where we may choose $x \geq 0$ as $A$ is positive. Similarly pick $y \in E_{+}$with $\|y\|=1$ and $\|B(y)\| \geq\|B\|-\epsilon$. Let $f \in E_{+}^{*}$ with $\|f\|=1$ and $f(B(y))=\|B(y)\|$. Take $T=f \otimes x$ which is positive with $\|T\|=\|T\|_{r}=\|f\| \times\|x\|=1$. As

$$
\left\|M_{A, B}(T)(y)\right\|=|f(B(y))|\|A(x)\| \geq(\|A\|-\epsilon)(\|B\|-\epsilon),
$$

we have $\left\|M_{A, B}\right\| \geq\|A\|\|B\|=\|A\|_{r}\|B\|_{r}$. From the preceding theorem, in general we have $\left\|M_{A, B}\right\|_{r}=\left\|\left|M_{A, B}\right|\right\|=\left\|M_{|A|,|B|}\right\|=\||A|\|\||B|\|=\|A\|_{r}\|B\|_{r}$. 


\section{THE OPERATOR NORM}

In this section, not only do we establish that we need not have $\left\|M_{A, B}\right\|=$ $\|A\|_{r}\|B\|_{r}$ (even in the setting of Corollary 3.2), but that there need not even be a constant $\alpha>0$ such that $\left\|M_{A, B}\right\| \geq \alpha\|A\|_{r}\|B\|_{r}$ for all $A, B \in \mathcal{L}^{r}(E)$. Our example will be on the space $\ell_{2}$ and will be built from finite dimensional examples. We need to estimate the operator norms, $\|A\|$, and regular norms, $\|A\|_{r}$, of certain square matrices $A=\left(a_{i j}\right)_{i, j=1}^{m}$ when considered as acting as linear operators on an $m$-dimensional Hilbert space $\ell_{2}^{m}$. We write $A_{i}=\sum_{j=1}^{m} a_{i j}$ for the sum of the $i$ 'th row of $A$ and then set $c_{j}=\sum_{i=1}^{m} a_{i j} A_{i}$.

Proposition 4.1. If $A$ is a $m \times m$ matrix, then

(1) $\frac{1}{m} \sum_{j=1}^{m} c_{j}=\frac{1}{m} \sum_{i=1}^{m} A_{i}^{2}=\frac{1}{m}\left\|\left(A_{i}\right)_{i=1}^{m}\right\|_{2}^{2} \leq\|A\|^{2}$.

(2) If $A$ has non-negative entries, then also $\|A\|^{2} \leq \max \left\{c_{j}: 1 \leq j \leq m\right\}$.

Proof. For (1) set $x=\left(\frac{1}{\sqrt{m}}\right)_{1}^{m}$ so that $\|x\|_{2}=1$ and $A x=\frac{1}{\sqrt{m}}\left(A_{1}, A_{2}, \ldots, A_{m}\right)$ so that $\|A x\|_{2}^{2}=\frac{1}{m} \sum_{i=1}^{m} A_{i}^{2}$, showing that $\|A\|^{2} \geq \frac{1}{m} \sum_{i=1}^{m} A_{i}^{2}$. Note also that

$$
\begin{aligned}
\sum_{j=1}^{m} c_{j} & =\sum_{j=1}^{m}\left(\sum_{i=1}^{m} a_{i j} A_{i}\right) \\
& =\sum_{i=1}^{m}\left(\sum_{j=1}^{m} a_{i j}\right) A_{i}=\sum_{i=1}^{m} A_{i}^{2} .
\end{aligned}
$$

To establish (2) we need to estimate $\|A x\|_{2}$ for $x \in \ell_{2}^{m}$ with $\|x\|_{2} \leq 1$ and (as $A$ has non-negative entries) all $x_{i} \geq 0$.

$$
\begin{aligned}
\|A x\|_{2}^{2} & =\sum_{i=1}^{m}\left(\sum_{j=1}^{m} a_{i j} x_{j}\right)^{2}=\sum_{i=1}^{m}\left[\sum_{j=1}^{m} a_{i j}^{2} x_{j}^{2}+2 \sum_{1 \leq j<k \leq m}^{m} a_{i j} a_{i k} x_{j} x_{k}\right] \\
& \leq \sum_{i=1}^{m}\left[\sum_{j=1}^{m} a_{i j}^{2} x_{j}^{2}+\sum_{1 \leq j<k \leq m}^{m} a_{i j} a_{i k}\left(x_{j}^{2}+x_{k}^{2}\right)\right]=\sum_{i=1}^{m}\left[\sum_{j=1}^{m}\left(\sum_{k=1}^{m} a_{i j} a_{i k} x_{j}^{2}\right)\right] \\
& =\sum_{i=1}^{m}\left[\sum_{j=1}^{m} a_{i j}\left(\sum_{k=1}^{m} a_{i k}\right) x_{j}^{2}\right]=\sum_{i=1}^{m}\left[\sum_{j=1}^{m} a_{i j} A_{i} x_{j}^{2}\right]=\sum_{j=1}^{m}\left[\sum_{i=1}^{m} a_{i j} A_{i}\right] x_{j}^{2} \\
& =\sum_{j=1}^{m} c_{j} x_{j}^{2} \leq \max \left\{c_{j}: 1 \leq j \leq m\right\} \sum_{j=1}^{m} x_{j}^{2} \leq \max \left\{c_{j}: 1 \leq j \leq m\right\}
\end{aligned}
$$

so that $\|A\|^{2} \leq \max \left\{c_{j}: 1 \leq j \leq m\right\}$.

Corollary 4.2. If $A$ is an $m \times m$ matrix of non-negative reals and each row of $A$ is constant, then

$$
\|A\|^{2}=\frac{1}{m} \sum_{i=1}^{m} A_{i}^{2}=\frac{1}{m}\left\|\left(A_{i}\right)_{i=1}^{m}\right\|_{2} .
$$

Proof. This follows immediately as all the $c_{j}$ are the same. 
Corollary 4.3. If $A$ is any real $m \times m$ matrix with each row constant, then

$$
\|A\|_{r}^{2}=\frac{1}{n} \sum_{i=1}^{m} A_{i}^{2}
$$

Proof. If $B=|A|$ and $B_{i}$ is the sum of the $i$ 'th row of $B$, then $B_{i}=\left|A_{i}\right|$ so that $B_{i}^{2}=A_{i}^{2}$. $B$ has constant rows so that

$$
\|A\|_{r}=\|B\|=\frac{1}{m} \sum_{i=1}^{m} B_{i}^{2}=\frac{1}{m} \sum_{i=1}^{m} A_{i}^{2}=\frac{1}{m}\left\|\left(A_{i}\right)_{i=1}^{m}\right\|_{2}^{2} .
$$

Define a sequence of matrices inductively by $K_{0}=(1)$ and

$$
K_{n+1}=\left(\begin{array}{cc}
K_{n} & K_{n} \\
-K_{n} & K_{n}
\end{array}\right) .
$$

These matrices were introduced to the study of positive operators by Krengel in 3 and [4. The matrix $2^{-n / 2} K_{n}$ is an orthogonal matrix so that as an operator on $\ell_{2}^{2^{n}}$ it is an isometry. It follows that $\left\|K_{n} x\right\|_{2}=2^{n / 2}\|x\|_{2}$ for all $x \in \ell_{2}^{2^{n}}$ and in particular $\left\|K_{n}\right\|=2^{n / 2}$ whilst it is easily checked, and well known, that $\left\|K_{n}\right\|_{r}=\left\|\left|K_{n}\right|\right\|=2^{n}$. In particular, we will make use of the fact that $\left|K_{n}\right|$ is the $2^{n} \times 2^{n}$ matrix with all its entries equal to 1 .

Our intention is to estimate the norm of the operators $M_{K_{n},\left|K_{n}\right|} \in \mathcal{L}\left(\mathcal{L}^{r}\left(\ell_{2}^{2^{n}}\right)\right)$ defined by $M_{K_{n},\left|K_{n}\right|}(T)=K_{n} T\left|K_{n}\right|$.

Example 4.4. Although $\left\|K_{n}\right\|_{r}=\left\|\left|K_{n}\right|\right\|=2^{n},\left\|M_{K_{n},\left|K_{n}\right|}\right\| \leq 2^{3 n / 2}$.

Proof. Take an arbitrary real $2^{n} \times 2^{n}$ matrix $T$. All the entries in the $i$ 'th row of $T\left|K_{n}\right|$ are equal to $T_{i}$, the sum of the $i$ 'th row in $T$. The columns in $K_{n} T\left|K_{n}\right|$ are therefore all equal to the product of $K_{n}$ with the column vector $\left(T_{i}\right)_{i=1}^{2^{n}}$ so that the column vectors of row sums for $K_{n} T\left|K_{n}\right|$ is precisely $2^{n} K_{n}\left(T_{i}\right)_{i=1}^{2^{n}}$. We may thus apply Corollary 4.3 to see that

$$
\begin{aligned}
\left\|K_{n} T\left|K_{n}\right|\right\|_{r}^{2} & =2^{-n} \times 2^{2 n}\left\|K_{n}\left(T_{i}\right)_{i=1}^{2^{n}}\right\|_{2}^{2} \\
& =2^{n}\left\|K_{n}\left(T_{i}\right)_{i=1}^{2^{n}}\right\|_{2}^{2} \\
& =2^{n}\left(2^{n / 2}\left\|\left(T_{i}\right)_{i=1}^{2^{n}}\right\|_{2}\right)^{2} \\
& =2^{2 n}\left\|\left(T_{i}\right)_{i=1}^{2^{n}}\right\|_{2}^{2},
\end{aligned}
$$

whilst by Proposition 4.1, $\|T\|_{r}^{2} \geq\|T\|^{2} \geq 2^{-n}\left\|\left(T_{i}\right)_{i=1}^{2^{n}}\right\|_{2}^{2}$, so that $\left\|M_{K_{n},\left|K_{n}\right|}(T)\right\|_{r}^{2}$ $=\left\|K_{n} T\left|K_{n}\right|\right\|_{r}^{2} \leq 2^{3 n}\|T\|_{r}^{2}$ and $\left\|M_{K_{n},\left|K_{n}\right|}\right\|_{r} \leq 2^{3 n / 2}$.

Embedding each of the examples on $\ell_{2}^{2}$ into $\mathcal{L}^{r}\left(\ell_{2}\right)$ shows that for regular operators $A, B$ on $\ell_{2}$ there is no real $\alpha>0$ such that $\left\|M_{A, B}\right\| \geq \alpha\|A\|_{r}\|B\|_{r}$.

\section{ACKNOWLEDGMENTS}

The author would like to thank Chen Jinxi for pointing out an error in the proof of Theorem 3.1 in a previous version of this paper. He would also like to thank Anton Schep for providing a correction for that error and for pointing out that Theorem 3.1 is essentially stated in the remark following Hilfsatz 3 in 7 . 


\section{REFERENCES}

[1] G. J. H. M. Buskes, P. G. Dodds, B. de Pagter, and A. R. Schep, Up-down theorems in the centre of $\mathcal{L}_{b}(E, F)$, Nederl. Akad. Wetensch. Indag. Math. 48 (1986), no. 1, 1-9. MR.834315 (87f:47052)

[2] Brian Jefferies and Susumu Okada, An operator bound related to regular operators, Arch. Math. (Basel) 66 (1996), no. 3, 219-227, DOI 10.1007/BF01195708. MR1373249 (97a:47042)

[3] Ulrich Krengel, Über den Absolutbetrag stetiger linearer Operatoren und seine Anwendung auf ergodische Zerlegungen (German), Math. Scand. 13 (1963), 151-187. MR0176034 (31 \#310)

[4] Ulrich Krengel, Remark on the modulus of compact operators, Bull. Amer. Math. Soc. 72 (1966), 132-133. MR0190752 (32 \#8162)

[5] Peter Meyer-Nieberg, Banach lattices, Universitext, Springer-Verlag, Berlin, 1991. MR.1128093 (93f:46025)

[6] Anton R. Schep, A remark on the uniform zero-two law for positive contractions, Arch. Math. (Basel) 53 (1989), no. 5, 493-496, DOI 10.1007/BF01324724. MR1019163 (90j:47046)

[7] J. Synnatzschke, Der Operator ${ }_{B} T_{C}: A \rightarrow B A C$ als vollstetiger Fastintegraloperator, Wiss. Z. Karl-Marx-Univ. Leipzig Math.-Natur. Reihe 24 (1975), no. 5, 529-534. Beiträge zur Theorie nichtnormierbarer topologischer Algebren. MR 0428103 (55 \#1132)

[8] J. Synnatzschke, Über einige verbandstheoretische Eigenschaften der Multiplikation von Operatoren in Vektorverbänden (German), Math. Nachr. 95 (1980), 273-292, DOI 10.1002/mana.19800950125. MR592901 (82b:47048)

[9] A. W. Wickstead, The centre of spaces of regular operators, Math. Z. 241 (2002), no. 1, 165-179, DOI 10.1007/s002090200411. MR1930989 (2004b:47069)

Pure Mathematics Research Centre, Queen's University Belfast, Belfast Bt7 1NN, NORTHERN IRELAND

E-mail address: A.Wickstead@qub.ac.uk 